\title{
Accurate assessment of computed order tracking
}

\author{
P.N. Saavedra and C.G. Rodriguez* \\ Department of Mechanical Engineering, University of Concepcion, Concepcion, Chile
}

Received 16 November 2004

Revised 4 July 2005

\begin{abstract}
Spectral vibration analysis using the Fourier transform is the most common technique for evaluating the mechanical condition of machinery working in stationary regimen. However, machinery operating in transient modes, such as variable speed equipment, generates spectra with distinct frequency content at each time, and the standard approach is not directly applicable for diagnostic. The "order tracking" technique is a suitable tool for analyzing variable speed machines. We have studied the computed order tracking (COT), and a new computed procedure is proposed for solving the indeterminate results generated by the traditional method at constant speed. The effect on the accuracy of the assumptions inherent in the COT was assessed using data from various simulations. The use of these simulations allowed us to determine the effect on the overall true accuracy of the method of different user-defined factors: the signal and tachometric pulse sampling frequency, the method of amplitude interpolation, and the number of tachometric pulses per revolution. Tests on real data measured on the main transmissions of a mining shovel were carried out, and we concluded that the new method is appropriate for the condition monitoring of this type of machine.
\end{abstract}

Keywords: Computed Order Tracking (COT), Variable speed machine diagnosis

\section{Introduction}

Industry is vertiginously evolving so as to adapt itself to ever more competitive markets. The observed trend is one of increasing rates of production, with higher quality, and lower costs. For these reasons, new machinery is bigger, more productive, and more complex. In production machines, unscheduled shutdowns generate high losses due to production downtime and maintenance costs. Because of this, maintenance strategy has changed from being a time-based preventive strategy to one of a condition-based predictive strategy.

Predictive maintenance monitors use certain measured or calculated parameters of the user's machinery that continuously determine the mechanical condition of the machine. This allows the user to carry out machine reparations only when the analysis of the monitored parameters indicates that it is necessary to do so. Early fault detection allows the user to repair a machine before a catastrophic fault occurs, and also allows the user to maintain a minimum stock of spares, because maintenance staff have a longer period of time over which to acquire spare parts.

Predictive maintenance uses various technologies to assess the condition of a machine. Of these techniques, vibration measurement and analysis have proven to be the most versatile and fundamental techniques for use in predictive maintenance. Any degradation of the mechanical conditions within a machine generates dynamic forces that change its vibration patterns. Using the proper processing technique, vibration analysis can identify specific degradation of machine components before any serious damage results.

${ }^{*}$ Corresponding author: C.G. Rodriguez, Casilla 160-C, Concepcion, Chile. Tel.: +56 41 204327; Fax: +56 41 251142; E-mail: psaavedr@ udec.cl; crrodrig@udec.cl. 
Spectral analysis using the standard fast Fourier transform approach, FFT, is the basic conventional technique used for evaluating the mechanical condition of machinery working in stationary and pseudo-stationary regimens. The FFT method is not directly applicable to the analysis of vibrations originating in non-stationary machines working at variable velocities. Consequently, non-stationary vibration analysis is a problem. This problem is present in certain critical machines, such as diesel motors, electromechanical shovel transmissions, and drills.

Order tracking spectrum and time-frequency transforms are techniques that have been used recently to solve the non-stationary vibration analysis problem. Order tracking spectrum [1-3], OTS, is a frequency analysis method that relates the spectral components of a vibration signal to the instantaneous rotational speed of a shaft, instead of to an absolute frequency base obtaining the also-called order spectrum, with the "order" defined as the frequency normalized by the shaft speed.

Time-frequency transforms, TFT, are three-dimensional representations that show the signal energy distribution in the time-frequency domain $[4,5]$. The TFT allow to visualize the frequency and amplitude variations of the spectral components in the measurement time. They are adequate to diagnostic in the case that the spectrum components are few. However, if the vibration signal contains many spectral components and significant changes of the rotational speed take place while the measurements are taken, the processed data will be confusing and difficult to analyze.

An approach for solving that is to use a new Transform proposed by Saavedra and Gonzalez [6] called Revolutionorder transform, ROT, which uses the advantages of both order tracking analysis and time-frequency transform. Basically, the technique involves changing the signal sampled at constant time increments to a signal sampled at constant shaft angular increments and then processes it using any TFT. ROT transforms the signal from time domain to the revolution-order domain. It is say order tracking spectrum is simply a bi-dimensional representation of ROT, where the magnitude of each component at OTS is an average of its values during the measurement time.

Blough [7] and Bai et al. [8] present different methods that allow obtaining a tri-dimensional representation similar to ROT. Blough makes use of angular shaft position information similar to COT and ROT methods, but processes it using the time variant discrete Fourier transform, TVDFT. The TVDFT is defined as a discrete Fourier transform whose kernel varies as a function of time defined as by the rpm of the machine. Bai et al. propose a model-based method for tracking the orders of vibration signal. This technique exploits adaptive filtering based on the recursive least-square algorithm, where the problem is treated as the tracking of frequency-varying band pass signals.

Considering the following, COT analysis method was the technique selected in this work for diagnosing the mechanical condition of machines that operate with high rate variable speed as the mining shovels: i) this technique has not rate variable speed limitation if the shaft speed is accurately estimated, ii) it is not problem that the data be analyzed in post-processing, not in real time iii) COT analysis is a bi-dimensional representation similar FFT, which it is an advantage for the personnel who works in the industries predictive maintenance considering that the knowledge obtained during years interpreting FFT spectra obtained on constant speed machines, can be directly transferred to the machines that work in regime of variable speed iv) the disadvantages of COT of not allow us to evaluated the severity of each spectral component and not visualize how their frequencies are changing is not a problem for diagnostic.

This paper describes a COT analysis method. The different factors that affect the method's accuracy were evaluated using numeric simulations of non-stationary vibrations. The use of these simulations allowed for the evaluated factors alone being modified from one test to another. In addition, the method allows for a comparison of the computed results with experimental data. The evaluation is necessary in addition to assess different COT commercial software considering that their performance depends on the hypothesis in which they are based, in contrast with the FFT processing that being a standard algorithm, similar results are obtained in all commercial software. Finally, the order tracking analysis method was used to analyze the real non-stationary vibration measured on a mining shovel, concluding that the method is appropriate for the analysis of this type of vibration.

\section{Order tracking analysis}

Spectra measured on machines that work at a constant rotational speed are principally composed of spectral components defined at discrete frequencies, which are generally related to the rotating speed of the shaft. The diagnostic procedure assumes a linear behavior of the machine, and each individual spectral component, or a group 

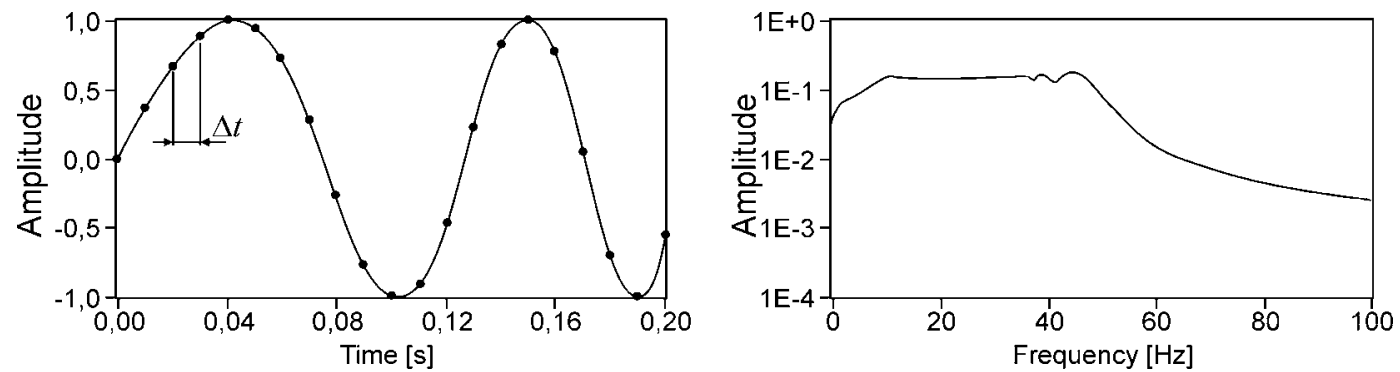

Fig. 1. Waveform and spectrum of a rotor's variable speed vibration sampled at constant increments of time.
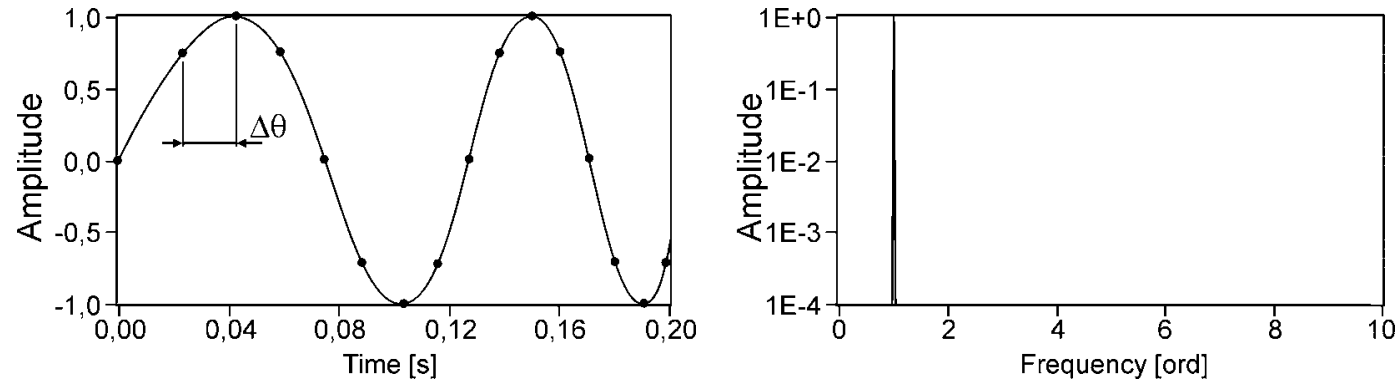

Fig. 2. Waveform and order spectrum of a rotor's variable speed vibration sampled using constant increments of shaft angle.

of them, is related to a specific dynamic force in the machine. These can be inherent in the operation of the machine, or can arise from a fault. However, when FFT analysis is applied to vibrations originating from machines working at variable rotational speeds, each spectral component spreads over several contiguous spectral lines, depending on the velocity changes in the machine. Because of this, it is impossible to analyze the resulting spectrum.

To illustrate this point, let us consider a machine with an unbalanced rotor. The vibration originating at a constant rotational speed of the machine is a sinusoidal vibration, with a frequency equal to the rotational speed of the machine. The FFT method will exhibit a unique discrete spectral component at the machine's operating speed. In contrast, if the machine's speed changes, then the spectrum shown in Fig. 1 is obtained. It can be seen that the spectral energy is spread over contiguous spectral lines according to the changes in speed, and that it is impossible to perform a diagnostic procedure. Figure 2 shows the spectral analysis of the previous vibration shown in Fig. 1, but using a COT method.

The order tracking analysis samples the vibration signal at constant increments in the shaft angle instead of constant increments of time, as is required by the FFT method. Once the signal is sampled at constant increments of the shaft angle, the FFT method can be applied to obtain an order spectrum. The order spectrum of the above signal vibration, as shown in Fig. 2, has a single frequency component at 1 ord or 1 xRPM, and is independent of the changes in velocity during data acquisition. This spectrum allows us to perform a diagnostic in a similar manner to that performed if the rotational speed remains constant.

There are at least two order spectrum methods that can be employed: the traditional hardware method, and the software computational method. The traditional hardware method directly samples the vibration signal at constant angle increments using analog instrumentation. The sampling frequency is fixed to track the shaft's rotational speed, and the shaft speed is calculated from two consecutive tachometric pulses. Ideally, this velocity could be used to fix the sampling frequency of the following shaft revolution. However, to adjust the antialiasing filter, the fixed sampling frequency is retarded for at least another revolution. Using a sampling frequency that was calculated two revolutions beforehand makes this method prone to error for a rapidly changing shaft speed. This has been pointed out by Tan and Mathew [1] and by Boosley et al. [2]. 


\section{Computed order tracking}

The COT technique records both the vibration signal and the tachometer pulse at constant time intervals using conventional hardware. The signal sampled at constant time intervals is resampled using a software package to obtain new data that are sampled at constant angular increments of the shaft's rotation. These new data are then processed using traditional FFT analysis. In this way, the frequency domain is changed to the order domain, as shown in Fig. 2.

The resampling procedure has two distinctive estimation steps:

1) The first is the determination of resample times at constant angle intervals, and

2) The second is an interpolation procedure used to estimate the amplitude of the vibration at the resample times.

\subsection{Traditional method}

The following section describes the COT technique documented by Fyfe and Munck [3].

\subsubsection{Determination of the resample times}

The vibration amplitude, $x(n \Delta t)$ for $n=0,1,2, \ldots$ is sampled at constant time increments using traditional hardware, as shown in Fig. 1. The COT analysis technique requires the resampling of the vibration amplitudes at constant angular increments, $\Delta \theta$, to obtain $x(k \Delta \theta)$, where $k=0,1,2, \ldots$ For this purpose, the first step is to determine the resample times, $t_{k}$, when the $k \Delta$ angles occur. In practice, a tachometer signal is usually available that can generate a known number of pulses per shaft revolution, (one pulse per revolution is the most common example). However, there is usually no information available to indicate the shaft position between these tachometer pulses. In this case, an interpolation method must be employed. At least three interpolation methods can be used: linear interpolation, quadratic interpolation, and cubic spline interpolation.

The simplest, and computationally cheapest, interpolation method is the linear interpolation. However, despite its simplicity, linear interpolation only produces acceptable results when the acceleration and deceleration of the shaft velocity are relatively low. A more accurate approach that has been used by several authors $[1,2]$ consists of assuming a constant angular acceleration of the shaft between tachometer pulses. Using this assumption, the angular shaft position, $\theta(t)$, is described by a quadratic equation:

$$
\theta(t)=b_{0}+b_{1} t+b_{2} t^{2}
$$

The cubic spline interpolation method generates a series of cubic polynomials to fit the data, and guarantees that up to the second derivative of the resulting function are continuous. In Section 5.2, we show that the use of cubic spline interpolation does not improve the accuracy of the results, and does not justify the higher computational cost.

In this paper, the angular shaft position at any given time is obtained by assuming a constant angular acceleration between tachometer pulses. This approach has been used by several authors [1-3]. Because the arrival times for three successive pulses, $t_{1}, t_{2}$, and $t_{3}$, and their respective angles, $\theta_{1}, \theta_{2}$, and $\theta_{3}$, are known, the polynomial coefficients $b_{0}, b_{1}$, and $b_{2}$ in Eq. (1) can be determined.

Since $\theta(t)$ is known between tachometer pulses, then it is possible to calculate the corresponding time for each constant angular increment, $k \Delta \theta$, and so solve Eq. (1) for a given value of $t$. Equation (1) has two possible solutions for $t$. In Appendix A, we show that the solution we need to consider is:

$$
t_{k}=\begin{gathered}
1 \\
2 b_{2}
\end{gathered}\left(\sqrt{ } 4 b_{2}\left(k \Delta \theta-b_{0}\right)+b_{1}^{2}-b_{1}\right)
$$

From Eq. (2), the time $t_{k}$, corresponding to any given constant shaft angular increment, $k \Delta \theta$, can be determined. When a new tachometer pulse arrives, the three previous pulses are used to determine a new quadratic polynomial. To avoid any overlap between adjacent polynomials, each local polynomial is defined over the central part of the interval,

$$
\begin{gathered}
\pi \\
N_{p}
\end{gathered} \leqslant k \Delta \theta<\begin{aligned}
& 3 \pi \\
& N_{p}
\end{aligned},
$$

where $N_{p}$ is the number of pulses per shaft revolution. 


\subsubsection{Determination of the amplitude of the resampled signal}

Once the resample times for constant angle increments are calculated, the corresponding amplitude at these times can be determined by interpolation between the original sampled data. There are different interpolation methods that can be used. Some of these methods have been described by Boosley et al. [2]. In Section 5.4, the linear and cubic spline interpolations, the most common current methods employed, are evaluated. Once the amplitude at the resampled times is estimated, then the resampled angle domain signal is obtained, and the angle domain signal is transformed to an order domain signal using the classical FFT approach.

\subsection{Proposed method}

The traditional method involves an inconvenient determination of the resample time $t_{k}$ using Eq. (2) for a constant rotational speed. In this case, $\theta(t)$ varies linearly, and the coefficient $b_{2}$ is zero. Therefore, the values of $t_{k}$ calculated using Eq. (2) become indeterminate. To avoid this inconvenience, we proposed the following modification to the traditional method. Instead of determining the corresponding time for each constant angular increment $k \Delta \theta$ as in the traditional method, we proposed to determine these times using Eq. (3), where the shaft angle position, $\theta_{n}$, corresponding to the original sample times $n \Delta t$, for $n=0,1,2, \ldots$ is

$$
\theta_{n}(t)=b_{0}+b_{1} n \Delta t+b_{2}(n \Delta t)^{2}
$$

We obtain a set of points, $\left(x_{n}, \theta_{n}\right)$, for each original sample time, $n \Delta t$. The value of the amplitude, $x_{k}$, corresponding to a constant angle increment, $\theta_{k}$, is determined by interpolation between the original data. A comparison between the traditional and proposed methods is given in Appendix B.

\section{Sources of error in computed order tracking}

The estimations made, and the inaccuracies inherent in digital signal processing are the main sources of error in the COT method. The estimations that affect the method's accuracy are:

1) The estimation of $\theta_{n}$ at constant time increments, $n \Delta t$.

2) The interpolations between the original data, $x_{n}$, to obtain the angle domain signal amplitudes, $x_{k}$.

\subsection{Errors in the estimation of the shaft angular position}

The first step in the proposed method is to determine the shaft angular position, $\theta_{n}$, corresponding to the original sample time, $t_{n}$. This determination is made assuming a constant angular acceleration, so that the shaft angle position, $\theta(t)$, can be calculated using Eq. (3). The accuracy of the calculated coefficients $b_{0}, b_{1}$, and $b_{2}$, depends on the accuracy of the determination of the pulse arrival times, as is discussed in the following subsections.

\subsubsection{Error due to the detection of the pulse arrival times}

The pulse arrival time is the moment when the tachometric pulse exceeds a threshold level, as shown in Fig. 3. The tachometric pulse and the vibration signal are discretely sampled. In Fig. 3, it is evident that a lower pulse sampling frequency means that a lower accuracy is obtained in the detection of the pulse arrival times. The simplest criterion to determine the pulse arrival time is to consider the first point above the threshold level. At low sampling frequencies, a way to improve the accuracy in the detection of the pulse arrival times is to consider the pulse arrival time as being the average between the points immediately above and immediately below the threshold level, as shown in Fig. 4. This allows the COT method's accuracy to increase, without increasing the computational costs.

\subsubsection{Errors due to variable angular acceleration}

Another source of error in determining the shaft angular position, $\theta(t)$, is due to the variation in angular acceleration, because the COT method assumes a constant shaft angular acceleration. If this assumption were not valid, then the determined shaft angle position and the resampled amplitude would be inaccurate, causing spectral noise. This is evaluated in Section 5.2. 


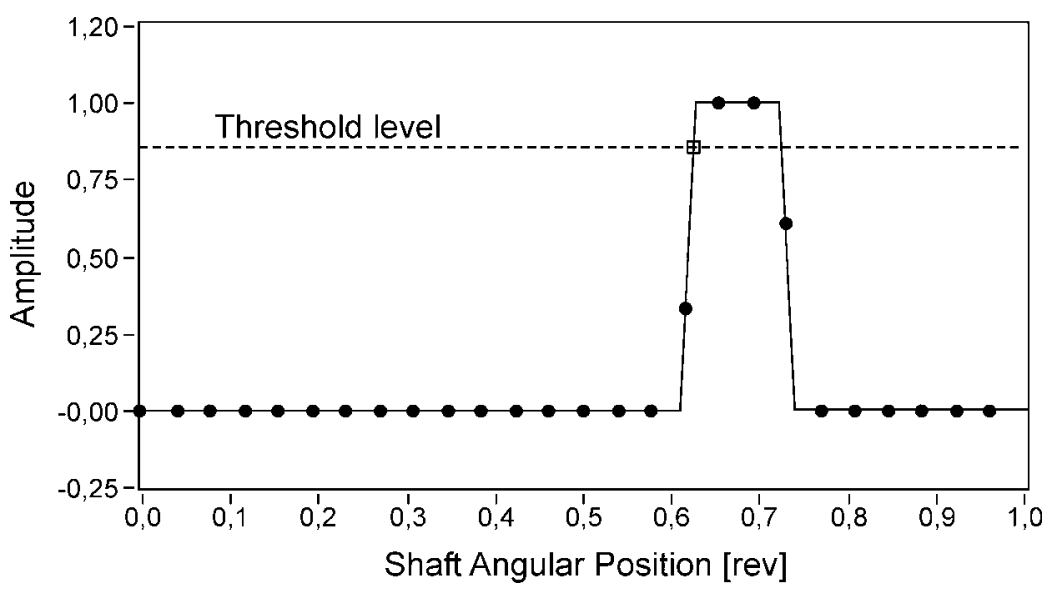

Fig. 3. Determination of the pulse arrival time.

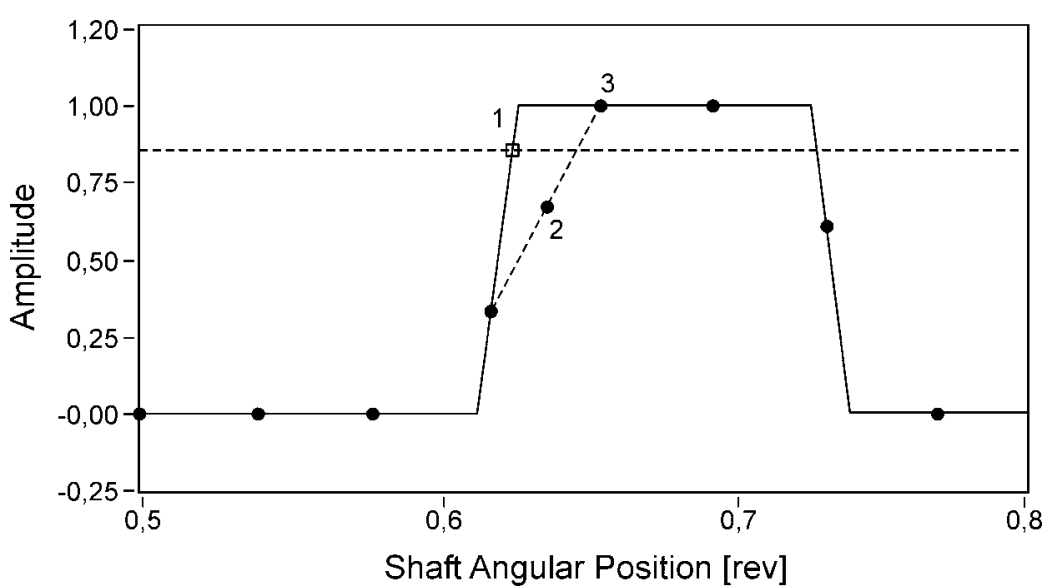

Fig. 4. Interpolation to improve the accuracy in the determination of the pulse arrival time. Key: $1=$ exact arrival time, $2=$ measured arrival time, and $3=$ averaged arrival time.

\subsection{Error in the estimation of resampled amplitude}

To determine the amplitude corresponding to the resample time at a constant angle increment, $\Delta \theta$, it is necessary to interpolate between the original sampled data for constant time increments. Figure 5 compares two popular interpolation methods: linear and cubic spline interpolations. From Fig. 5, it is clear that the interpolation method affects the method's accuracy only at low sampling frequencies. This effect is evaluated in Section 5.4.

\section{Performance evaluation of computed order tracking}

The COT performance was evaluated using simulated numerical signals. Numeric simulation has a great advantage, in that the exact signals in both the time and order domains are known, allowing for a comparison between true and calculated values. The simulations also allow us to individually evaluate the incidence of each error source.

The difference between the true signal and the resampled signal generates the noise floor in the COT spectrum. This fact makes it difficult to diagnose the mechanical condition of the machine. On the one hand, the spectral noise distributed in all the spectral lines can hide the low amplitude components of the vibration signal that are generated by an early fault. On the other hand, discrete noise spectral components that are above the noise floor, especially the ones that are synchronous with the machine speed, can induce errors in diagnosing the machine's condition. 


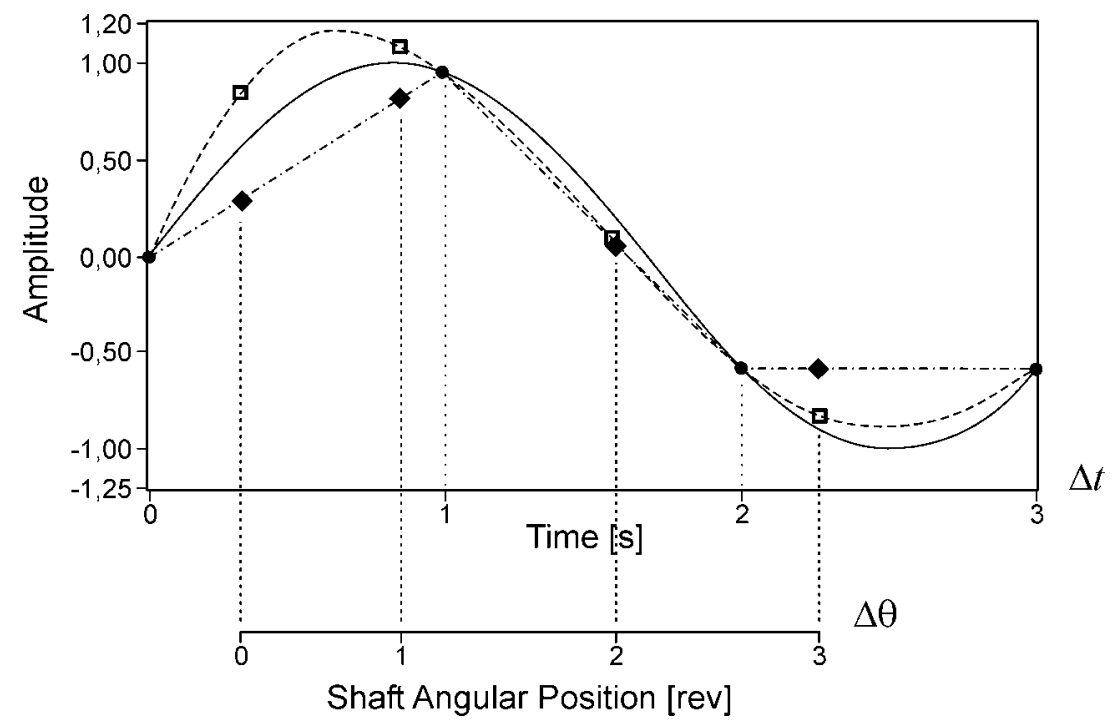

Fig. 5. Determination of the amplitude of the resampled signal. Key: $\downarrow=$ linear interpolation, $\square=$ cubic spline interpolation, and $\bullet=$ sampled data.

These two problems were evaluated using the following two indicators.

1) The noise percentage. The noise percentage, noise $\%$, was defined as the percentage of the rate between the RMS noise value and the RMS value of the true signal. This indicator is a global value that indicates the difference between the true signal and the resampled signal. For a signal of $N$ points, the noise percentage is given by:

$$
\text { noise percentage }=\frac{\sqrt{\sum_{i=1}^{N}(y(i)-\hat{y}(i))^{2}} \cdot 100 \%}{\sqrt{\sum_{i=1}^{N}(\hat{y}(i))^{2}}}
$$

where $y(t)$ is the exact signal and $\hat{y}(t)$ is the resampled signal.

2) The amplitude rate percentage. The amplitude rate percentage was defined as the percentage of the rate between the greatest noise spectral component and the exact signal amplitude.

There is no correlation between these two indicators, as they do not change in syntony. To illustrate this, let us consider a sinusoidal vibration with a constant amplitude and a frequency of 30 times the pulse frequency. If the error in the detection of the pulse arrival time is $1 / 120$ of the pulse period $\left(3^{\circ}\right)$, then the waveform of the true vibration and the resampled signal will be exactly the same, but with a phase difference of a quarter of the signal period $\left(3^{\circ} \times 30=90^{\circ}\right)$. The noise percentage will have a high value, but the amplitude rate percentage will be zero.

\subsection{Effect of the sampling frequency on COT accuracy}

The accuracy in determining the pulse arrival time depends on the pulse sampling frequency, as can be inferred from Fig. 3. As the pulse sampling frequency increases, then the accuracy in the determination of the arrival time increases. To quantify this effect, a sinusoidal vibration signal of amplitude $=1.0$ was simulated. Its frequency began at $10 \mathrm{~Hz}$, and increased at a constant rate of $10 \mathrm{~Hz} / \mathrm{s}$. At this point, the noise generated only by the timing inaccuracies of the tachometric pulse was evaluated. For this, the true amplitude value was used. Figure 6 shows the noise signal in the angle domain and its computed order spectrum for given pulse-sampling frequencies. In Fig. 6, it can be seen that the noise level decreases as the sampling frequency increases, and that the most significant noise component is at the 1 ord frequency with lateral sidebands. 

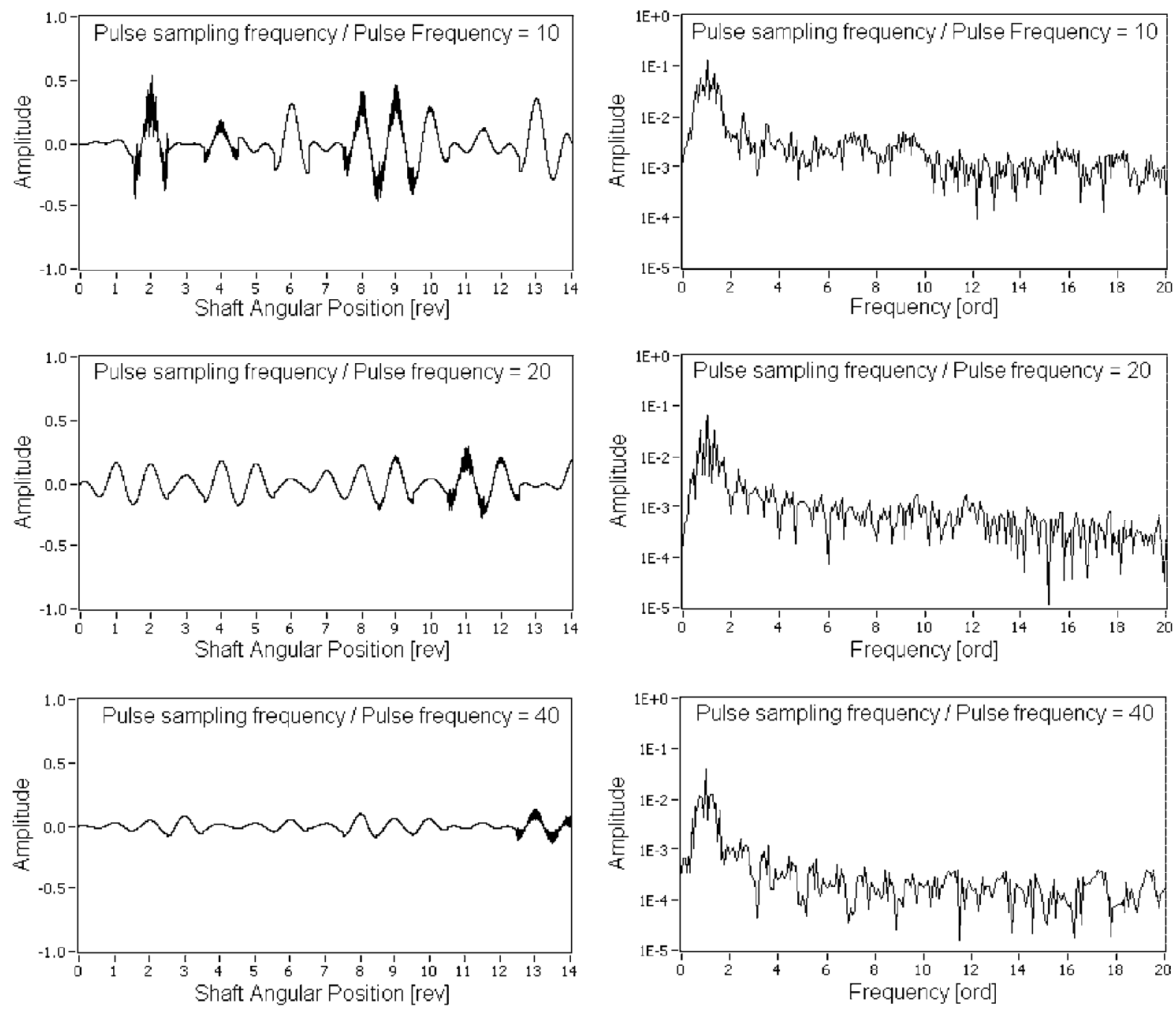

Fig. 6. Noise due to the pulse sampling frequency.

Figure 7 shows how the amplitude rate percentage and the noise percentage vary with the rate between the pulse sampling frequency and the pulse frequency. It can be seen that both noise indicators decrease as the sampling rate increases. Table 1 shows some discrete values obtained from these figures.

In practice, this data will be useful for determining the pulse sampling frequency required for a given accuracy. To determine the pulse sampling frequency it is necessary to consider that the FFT method requires the use of a weighting window. Windowing generates lateral leaks consisting of spectral lobes sited beside the discrete spectral components. In diagnostic practice, the weighting window most often used in FFT analysis is the Hanning window, which gives the best compromise between frequency and amplitude accuracy. The greatest spectral lobe generated using the Hanning window is at $2.5 \%$ ( $-32 \mathrm{~dB}$ ) of the amplitude of the spectral component. In this paper, this figure is considered to be an acceptable value of the noise generated by the COT.

The discrete values from Fig. 7 shown in Table 1 reveal that to obtain an amplitude rate percentage of the noise below $2.5 \%$ (i.e., an acceptable value of the noise), a pulse sampling frequency 35.3 times higher than the pulse frequency is required.

The above recommendation is valid for a 1 ord signal. For vibration signals of greater order, a higher pulse sampling frequency is required to obtain the same noise level as that of a 1 ord signal. For example, an error of 
Table 1

Noise levels for various pulse sampling frequencies

$\begin{array}{rcc}F_{m} & \text { Rate amplitude percentage } & \text { Noise percentage } \\ F_{\text {pulse }} & & \\ 2.0 & 65.2 & 583.9 \\ 3.0 & 34.0 & 532.0 \\ 4.0 & 25.3 & 449.9 \\ 6.0 & 15.8 & 257.6 \\ 10.0 & 9.5 & 144.6 \\ 20.0 & 3.6 & 84.6 \\ 35.3 & 2.5 & 42.4\end{array}$
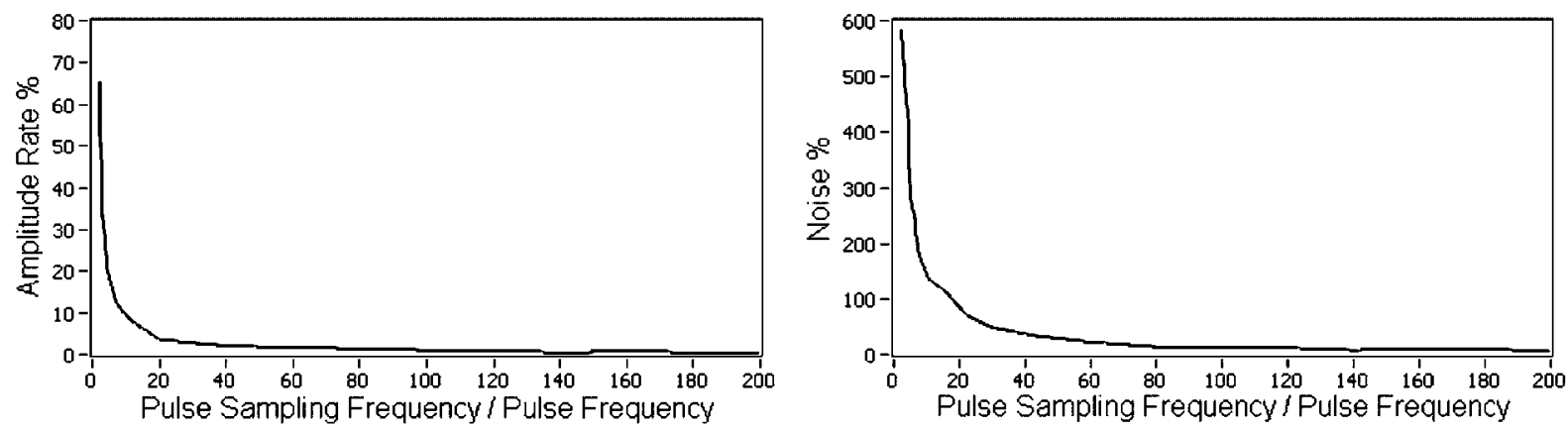

Fig. 7. Noise levels due to the pulse sampling frequency.
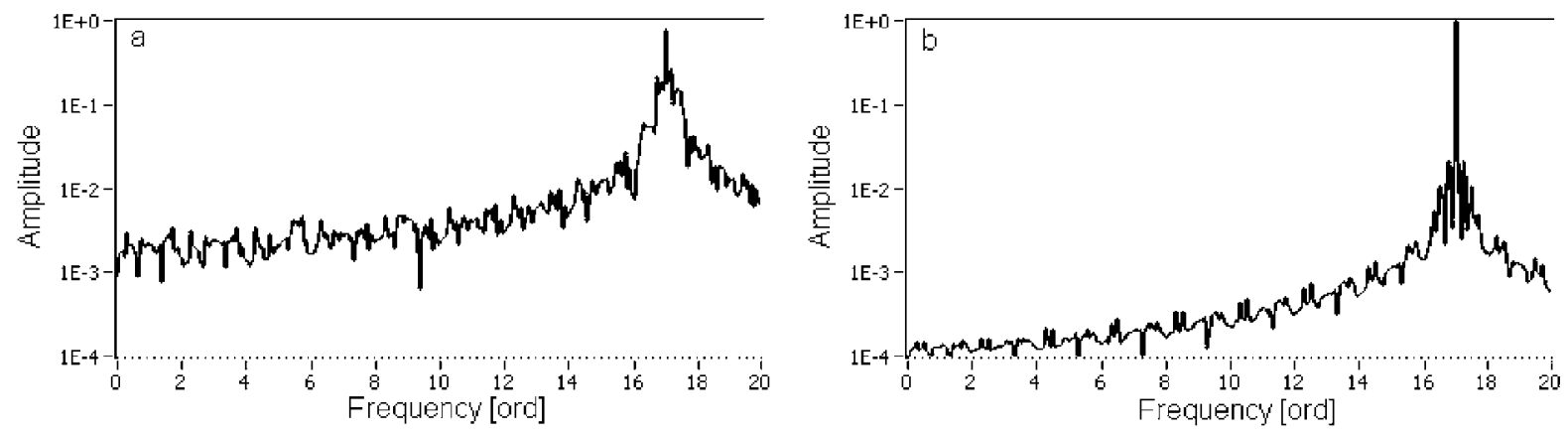

Fig. 8. Order spectrum of a 17 ord signal.

$0.01 \mathrm{rev}$ in the measurement of the pulse arrival time means a $1 \%$ in the 1 ord signal period, but a $20 \%$ in the 20 ord signal period. To obtain equivalent noise levels in a $Z$ ord signal as in a 1 ord signal, the pulse sampling frequency must be $Z$ times greater than the pulse sampling frequency for a 1 ord signal. Figure 8(a) shows the computed order spectrum of a 17 ord signal using a pulse sampling frequency of 35.3 times the pulse frequency. The greatest noise spectral component is $26.4 \%$ of the original signal, compared with $2.5 \%$ for the 1 ord signal. Figure 8 (b) shows the spectrum of a 17 ord signal using a pulse sampling frequency that was 600 times $(17 \times 35.3)$ the pulse frequency. The greatest noise spectral component is $2.4 \%$ of the original signal, which is similar to the noise level obtained for a 1 ord signal with a pulse sampling frequency of 35.3 ord.

\subsection{Varying shaft angular acceleration}

The condition of constant shaft angular acceleration does not occur frequently in real machines. There are some machines, however, that work under varying rotational speed conditions. An example of this is the hoist, crowd, and swing transmission in an electromechanical mining shovel. Figure 15 shows the varying rotational speeds measured in a hoist transmission motor during an ore charging cycle. 


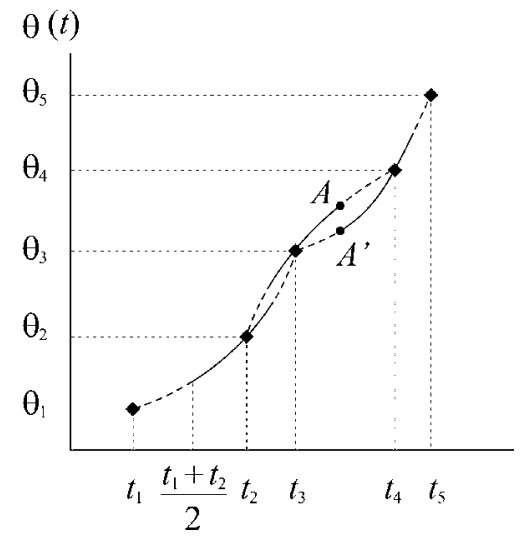

Fig. 9. Most unfavorable case for varying shaft acceleration. Key:

$=$ pulse arrival time.

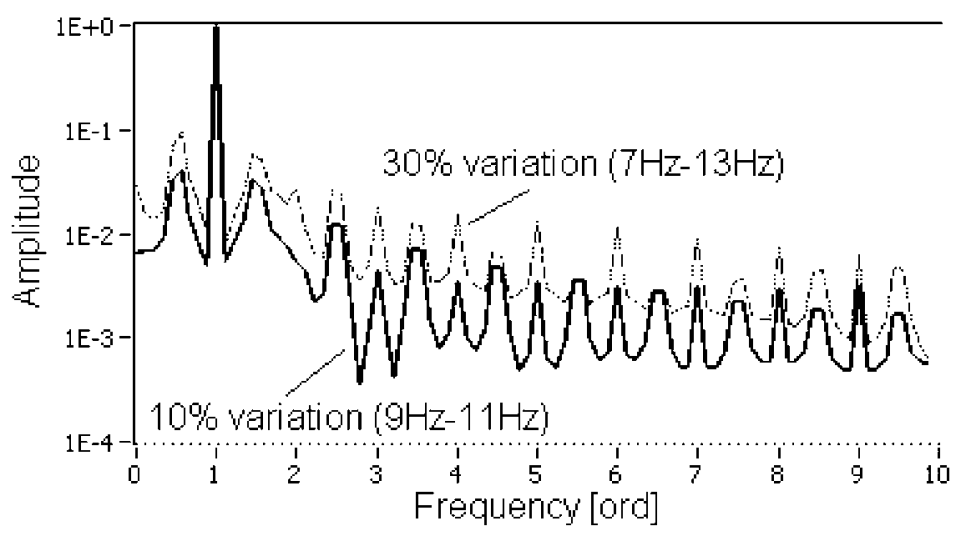

Fig. 10. Order spectrum with varying sinusoidal acceleration.

In the following discussion, we analyze the effect on the accuracy of the order spectrum when the assumption of constant acceleration does not hold. The shaft angle position, $\theta(t)$, is estimated from parabolic segments using Eq. (1). These segments are determined from three consecutive pulse arrival times, that is, once every two shaft revolutions (if one pulse per revolution is available). To avoid any overlap between adjacent parabolas, each local parabola is utilized only over the central part of the interval. Therefore, a new parabolic segment is utilized in each shaft revolution.

The most unfavorable condition for varying shaft acceleration is shown in Fig. 9. This condition occurs when the shaft's angular acceleration alternates from positive to negative during each shaft revolution. In this case, the parabolic segments alternate between concave and convex shapes, generating large discontinuities between two resampled adjacent angles $\theta_{k}$ that pertain to different segments. These are denoted by the letters $A$ and $A^{\prime}$ in Fig. 9 .

To quantify the effect on the COT accuracy when the assumption of constant acceleration is not valid, a simulated vibration was analyzed that only considered the effect of variable shaft acceleration. For this reason, we used the exact pulse arrival times and the exact vibration amplitude. The simulated vibration signal was a sinusoidal synchronous component of amplitude $=1.0$. The rotational speed varied as a $5 \mathrm{~Hz}$ sinusoidal waveform between 9 and $11 \mathrm{~Hz}$ around its mean value of $10 \mathrm{~Hz}$ (a variation of $10 \%$ ), which is the most unfavorable case inferred from Fig. 9.

The calculated order spectrum for this signal vibration is shown in Fig. 10. Note that a group of discrete spectral components with multiple frequencies of 0.5 ord was observed. The origin of these components is inferred by analyzing Fig. 11(a), which shows the generated noise (the difference between the true signal and the resampled signal). It can be seen that the waveform of the noise in the angle domain is periodic. The periodicity occurs over two 

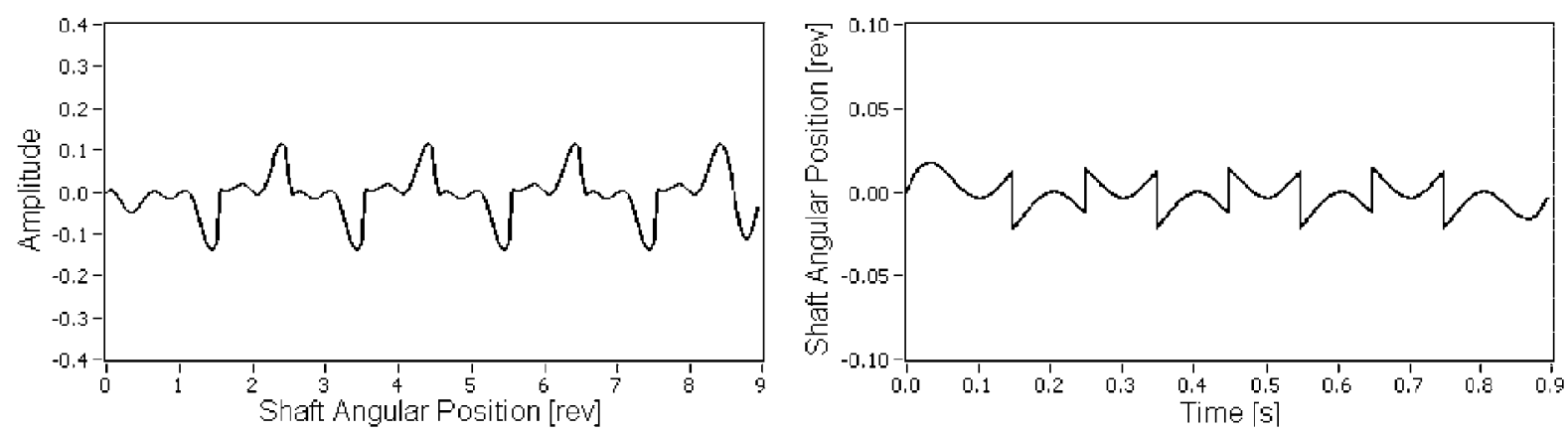

Fig. 11. Noise and difference between the resampled angle position, and the true angle position.

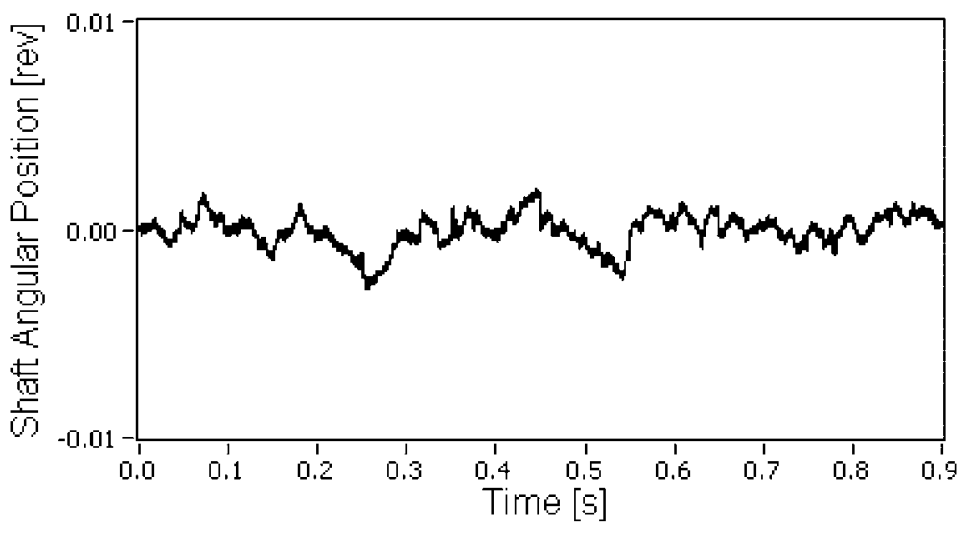

Fig. 12. Waveform of generated noise.

revolutions, or in a frequency of 0.5 ord. Figure 11(b) shows the difference between the resampled angle position, $\theta_{k}$, and the true angle position. It can be seen that large discontinuities occur of around $0.1 \mathrm{~s}$, corresponding to one shaft revolution. These abrupt discontinuities are generated, as shown graphically in Fig. 9, when the resampled points change from one parabolic segment to another parabolic segment.

In practice, it is not common to find such periodic variations of the rotational speed as were seen in the above case. It is more probable that the rotational velocity varies in a random mode. Such a case was analyzed using a simulated signal consisting of a synchronic sinusoidal vibration of amplitude $=1.0$. The rotational speed varied in a random mode between 9 and $11 \mathrm{~Hz}$ (a variation of 10\%) around its mean value of $10 \mathrm{~Hz}$. Figure 12 shows the waveform of the noise signal. It can be seen that the amplitude of the random signal is much smaller than the amplitude in the case discussed above. Figure 13 shows the order spectrum for the random simulated signal. This spectrum is composed of a discrete component at 1 ord, and of very small components distributed in all the spectral lines, typical of a random signal spectrum.

In the most unfavorable case shown if Fig. 10 that corresponds to the velocity changing in a sinusoidal pattern, the noise was generated by the large discontinuities between two resampled adjacent angles $\theta_{k}$ that pertained to different parabolas segments. One could suppose that it would be possible to minimize this inaccuracy by using a more sophisticated interpolation method, such as a cubic spline.

The order spectrum obtained using a cubic spline interpolation is shown in Fig. 14. This spectrum exhibits lower spectral components at the higher 0.5 ord harmonics than the spectrum shown in Fig. 10, which uses a quadratic interpolation for determining the shaft angular position. An improvement is seen in the low noise components (those below $1 \%$ of the signal amplitude), but not for the higher noise components. Therefore, for practical diagnostic purposes, the increase in accuracy of the method that utilizes a cubic spline interpolation for determining the shaft angular position instead of the quadratic interpolation is insignificant. 


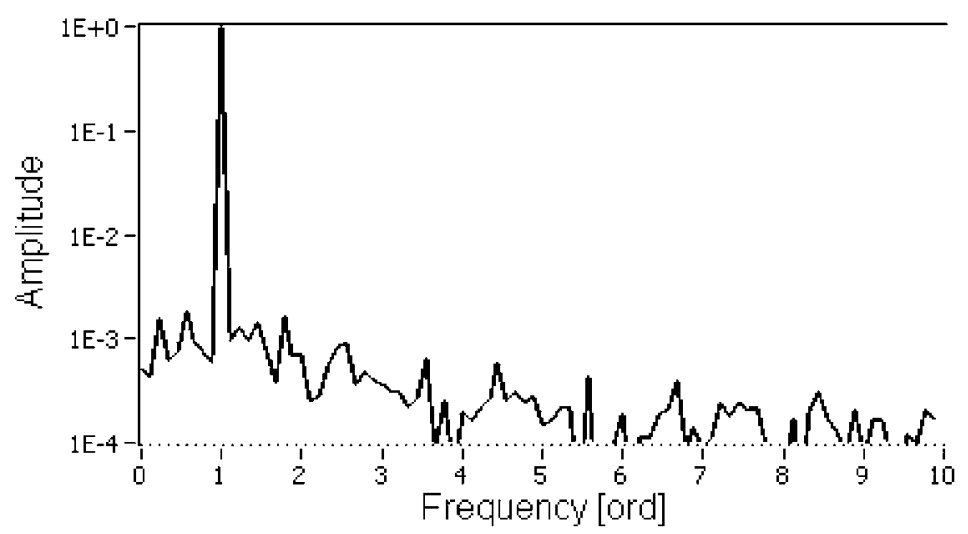

Fig. 13. Order spectrum of varying random acceleration.

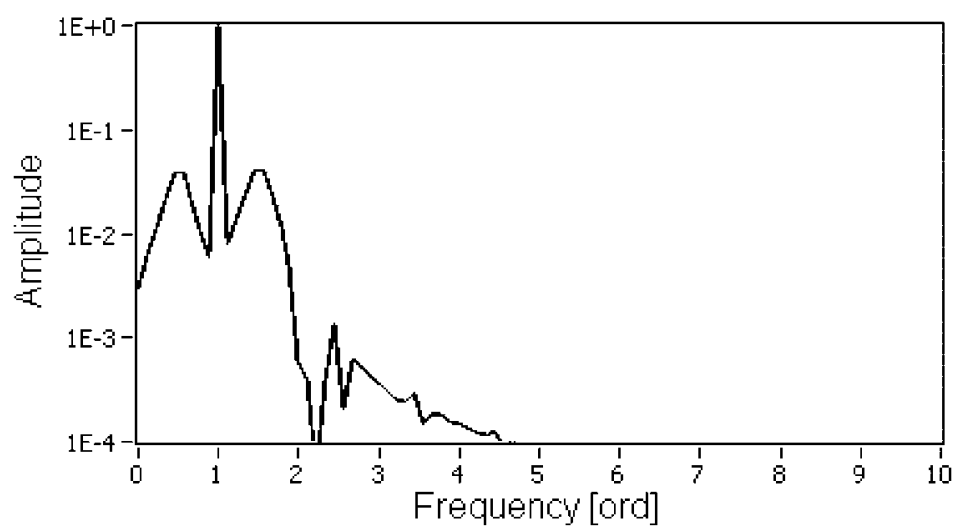

Fig. 14. Order spectrum for a varying sinusoidal acceleration and a spline interpolation of the shaft angular position.

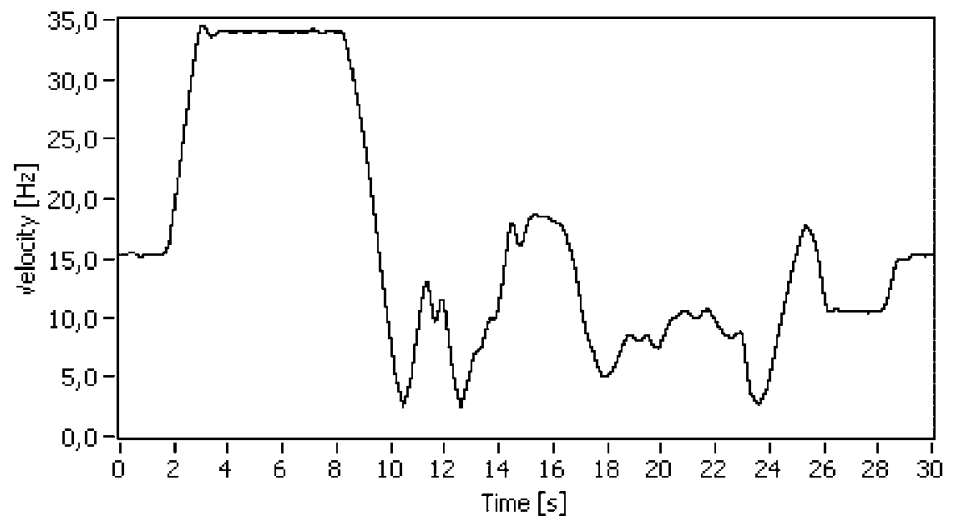

Fig. 15. Speed variation of the hoist transmission of a mining shovel.

The next simulated signal was that of a synchronic sinusoidal vibration with amplitude $=1.0$. This sinusoidal vibration is synchronic to the rotational speed varied as shown in Fig. 15. This rotational speed was measured on an electrical motor on the hoist transmission of a mining shovel. Figure 16 shows the order spectrum of the simulated signal. It can be seen that in spite of the hypothesis of constant angular acceleration not being satisfied, the noise 
Table 2

Noise levels for various linear variation of acceleration

Linear variation of acceleration $\left(\mathrm{Hz} / \mathrm{s}^{2}\right)$

Percentage

Max amplitude Noise

$0.000 \quad 0.00$

$0.009 \quad 0.16$

$0.012 \quad 0.19$

$0.024 \quad 0.39$

$0.048 \quad 0.77$

$0.074 \quad 1.12$

$0.076 \quad 1.55$

$\begin{array}{lll}15.0 & 0.074 & \\ 20.0 & 0.076 & 1.55 \\ 30.0 & 0.096 & 2.21\end{array}$

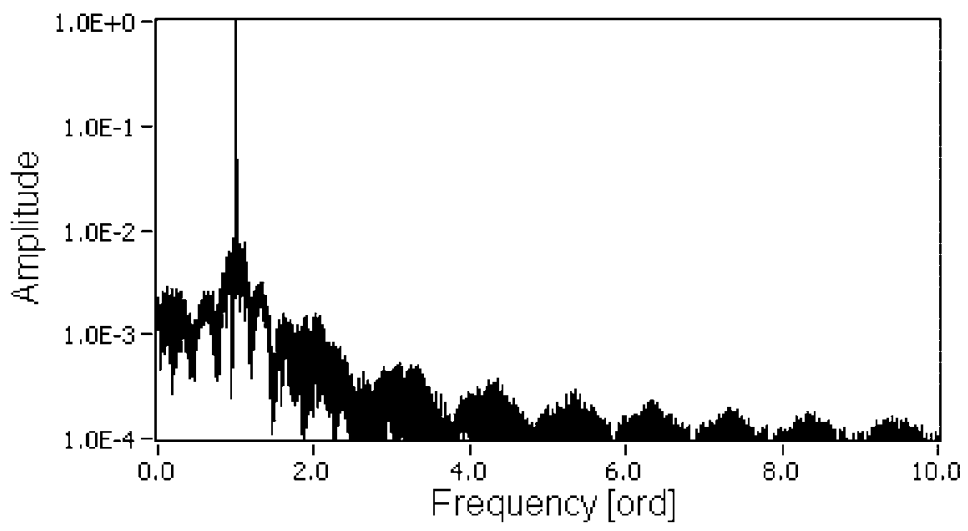

Fig. 16. Order spectrum of the simulated signal from a mining shovel.

level generated is insignificant. The amplitude rate percentage was only $1.08 \%$.

Another example where the noise generated was insignificant, when the assumption of constant acceleration was not hold, was during the run-up and run-down procedures of machines where the rotational speed did not vary in a linear mode. This can be qualitatively inferred from a graphical representation similar to that shown in Fig. 9. Table 2 summarizes some results obtained from numerical simulations assuming a quadratic variation of the rotational speed (linear variation of the angular acceleration) for various acceleration rates. From these results, it can be concluded that the noise generated is negligible (i.e., the amplitude rate percentages are below $0.1 \%$ ). This has been qualitatively pointed out by Fyfe et al. [3].

\subsection{Multiple tachometric pulses per revolution}

By simple inspection of Fig. 9, it is evident that if the number of pulses per shaft revolution is increased, then the discontinuities generated in passing the resampled points from one parabolic segment to another will decrease. As a consequence, the noise will decrease and the COT accuracy will increase. To quantify this effect, the order spectrum shown in Fig. 10 calculated using one pulse per revolution was recalculated using 12 pulses per revolution. Figure 17 shows the resulting order spectrum. It can be seen that the noise has significantly decreased: we obtained a negligible amplitude rate percentage of $0.02 \%$ and a noise level of $0.92 \%$. It must be kept in mind, however, that the simulated variation in rotation speed in this case was the most unfavorable for obtaining a high accuracy from the COT, as was indicated above.

\subsection{Amplitude interpolation}

As was previously pointed out, to determine the signal amplitude at the resample times, an interpolation between the amplitudes of the original sampled signal must be used. Looking at Fig. 5, it is evident that at a higher sampling 


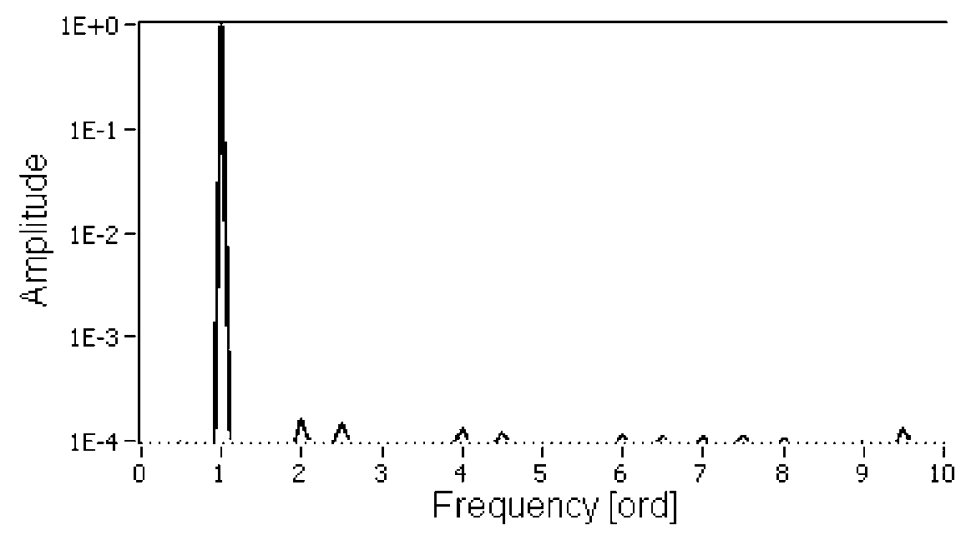

Fig. 17. Order spectrum using multiple pulses per revolution.

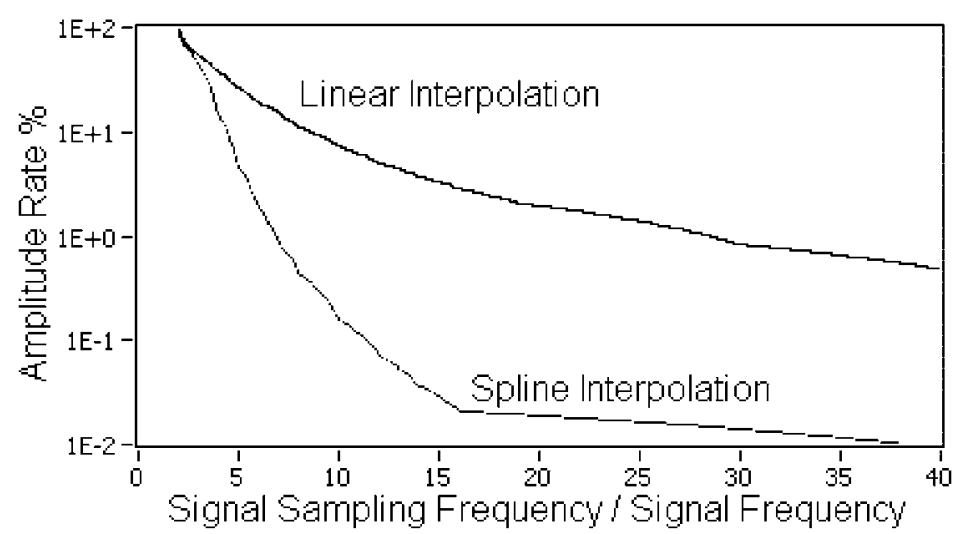

Fig. 18. Noise level for different sampling frequencies and interpolation.

frequency of the signal, a more accurate amplitude interpolation of the resampled signal occurs, and a more accurate COT is obtained. To quantize this effect, we simulated a sinusoidal vibration signal of amplitude $=1.0$. Its frequency began at $10 \mathrm{~Hz}$, and accelerated at a constant rate of $10 \mathrm{~Hz} / \mathrm{s}$.

The exact pulse arrival times were used to evaluate the effect of the amplitude interpolation on the method accuracy alone. Figure 18 shows the variation of the amplitude rate percentage with the rate between the signal sampling frequency and the signal frequency. It can be seen that this noise indicator decreased as the frequency rate increased, and that the noise level generated using a linear interpolation was higher than the noise level generated using a cubic spline interpolation for all frequency rates.

Table 3 shows some discrete values of the amplitude rate percentage that can be useful in choosing a signal sampling frequency to obtain a given noise level. It can be seen that an acceptable value of $2.5 \%$ for the amplitude rate percentage is obtained with a sampling frequency of 5.8 and 17.3 times the pulse frequency using a cubic spline interpolation and a linear interpolation, respectively.

\subsection{Combinated effect of the signal and pulse sampling frequency}

In Sections 5.1 and 5.3, several signals were simulated to evaluate the separate influences of the pulse and of the signal sampling frequency on the COT method's accuracy. In this section, the combined effect is quantified, that is, the pulse and vibration signal were sampled. The vibration signal simulated was a single sinusoidal signal of amplitude $=1.0$, whose frequency began at $10 \mathrm{~Hz}$ and accelerated at a constant rate of $10 \mathrm{~Hz} / \mathrm{s}$. Table 1 shows that for an infinite sampling frequency for the signal (where the exact amplitude is known), a pulse sampling frequency 
Table 3

Amplitude rate percentage

\begin{tabular}{ccc}
$F_{m}$ & \multicolumn{2}{c}{ Interpolation } \\
$F_{\mathrm{se}}$ & Linear & Spline \\
& 90.6 & 96.6 \\
2.0 & 94.5 & 45.2 \\
3.0 & 54.5 & 14.6 \\
4.0 & 37.6 & 2.5 \\
5.8 & 23.0 & 1.8 \\
6.0 & 19.0 & 0.16 \\
10.0 & 7.3 & 0.03 \\
17.3 & 2.5 & 0.02 \\
20.0 & 1.9 &
\end{tabular}

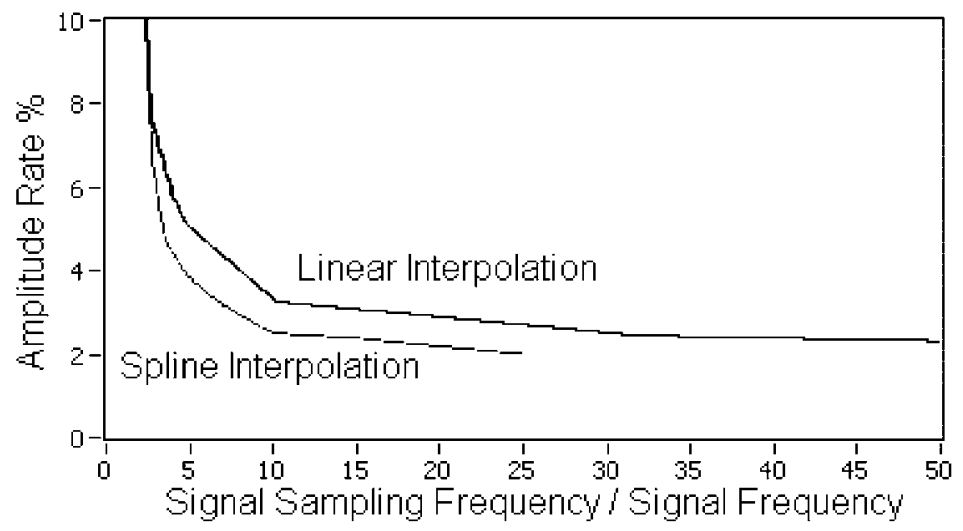

Fig. 19. Amplitude rate percentage for a pulse sampling frequency of 35.3 times the pulse frequency.

of 35.3 times the pulse frequency is required to obtain an amplitude rate percentage of $2.5 \%$. Analysis of the noise levels obtained using the same pulse sampling frequency of 35.3 times the pulse frequency for several signal sampling frequencies using the cubic spline and linear interpolation was carried out. Figure 19 shows the results obtained. It can be seen that for a signal sampling frequency of 29.4 and 10.6 times the signal frequency, the amplitude rate percentage is $2.5 \%$ for a linear interpolation and for a spline interpolation, respectively.

These results are useful in that they indicate that if a data acquisition system employing the same sampling frequency for the tachometric pulse and signal is used, then the sampling frequency required to obtain a given method accuracy is defined by the pulse requirements (see Table 1). In this case, there are no advantages in using an interpolation technique that is more accurate than the linear interpolation. This has been pointed out by Fyfe and Munck [3] and by Boosley et al. [2].

If the data acquisition system allows for sampling the pulse and signal with different frequencies, then it is recommended that the signal and pulse sampling frequencies be selected independently, in agreement with Tables 1 and 2 , respectively. In this case, it is also recommended to use the cubic spline interpolation, bearing in mind that the linear interpolation method requires a higher sampling frequency than the spline interpolation method to obtain a given accuracy. It must also be kept in mind, however, that a higher sampling frequency requires more computer resources for storing a larger quantity of raw data.

\section{Analysis of a real case}

In this point the technique previously described is used for analysing the vibration measured in a shovel that is a critical mining machinery.

The concept of using vibration analysis on shovels is not new. The mining industry implemented this strategy in the past, however, with different levels of success. It has been difficult until now to transfer the exact general 


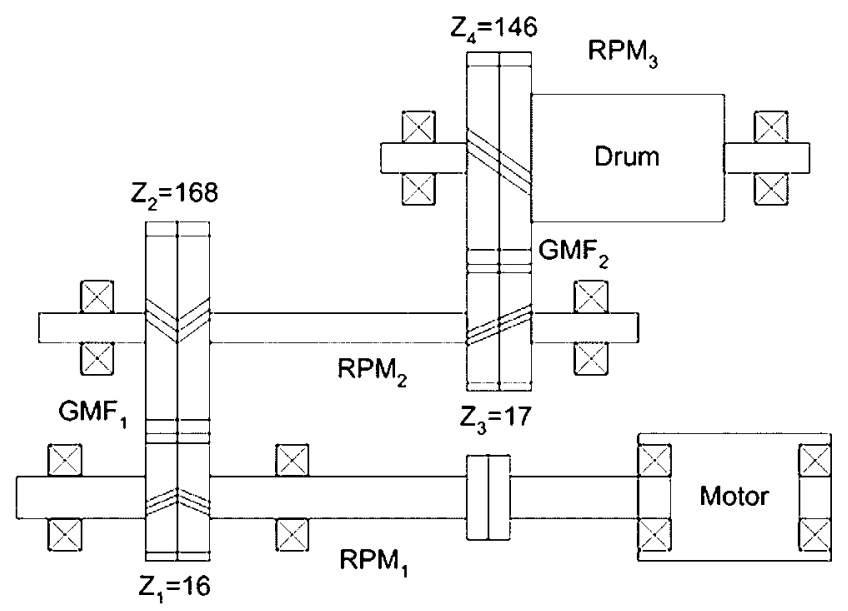

Fig. 20. Scheme of the shovel transmission.

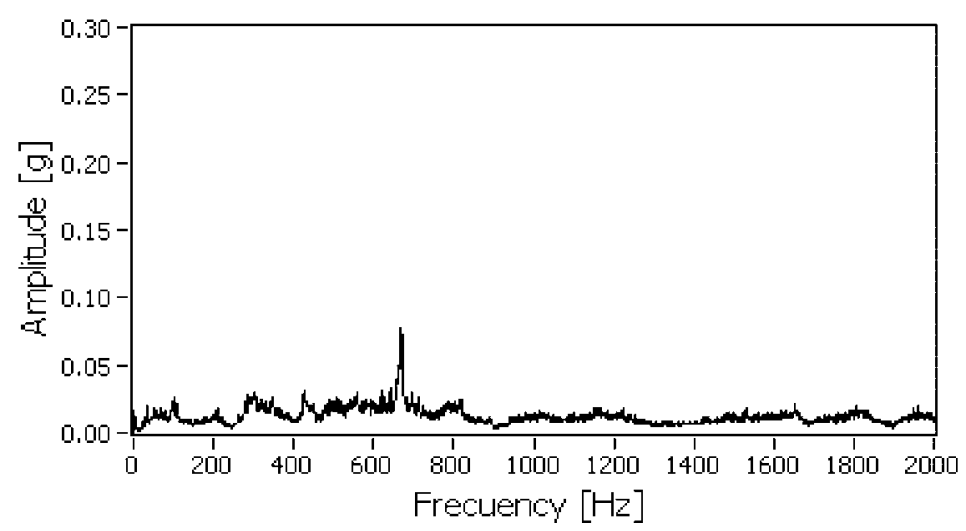

Fig. 21. Classical FFT spectrum.

industry analysis to the electromechanical mining shovels due to the fact that they operate in an intermittent and short duration of high rate variable speed condition.

The vibration monitoring in electromechanical shovels in the mining industry has traditionally been made in periodic form with the shovel out of production and the dipper empty, in order to obtain constant speed and load operation conditions. However, this method has the inconvenience of the significant loss of production while the vibration measurement is being taken.

The present challenge is to monitor the shovel when it is working. This point describes how the precedent technique can be used for implementing an efficient predictive maintenance strategy in the electromechanical mining shovels without loss of production. Figure 20 shows a scheme of the shovel transmission.

We will concentrate on the hoist transmission, which analysis is the most difficult considering the short working cycles and its high speed changes. The hoist transmission ascends and lowers the dipper through a system ropes and sheaves. The major components of the mechanism are: a variable speed and reversible AC motor; a first reduction consisting of a pair of double helicoidally gears with 16 and 168 teeth respectively; a second reduction of helicoidally gears with 17 and 146 teeth respectively and a drum containing the ropes directly coupled to the larger gear in the second gear set. The gear mesh frequency of the first reduction is GMF1 $=16 \times$ RPM1, and the gear mesh frequency of the second reduction is GMF2 $=17 \times \mathrm{RPM} 2=1.62 \times \mathrm{RPM} 1$.

Figures 21 and 22 shows the classical FFT spectrum and the computed order spectrum respectively of the horizontal vibration measured on the inboard bearing of the gear box during the time that the shovel is charging ore into its 


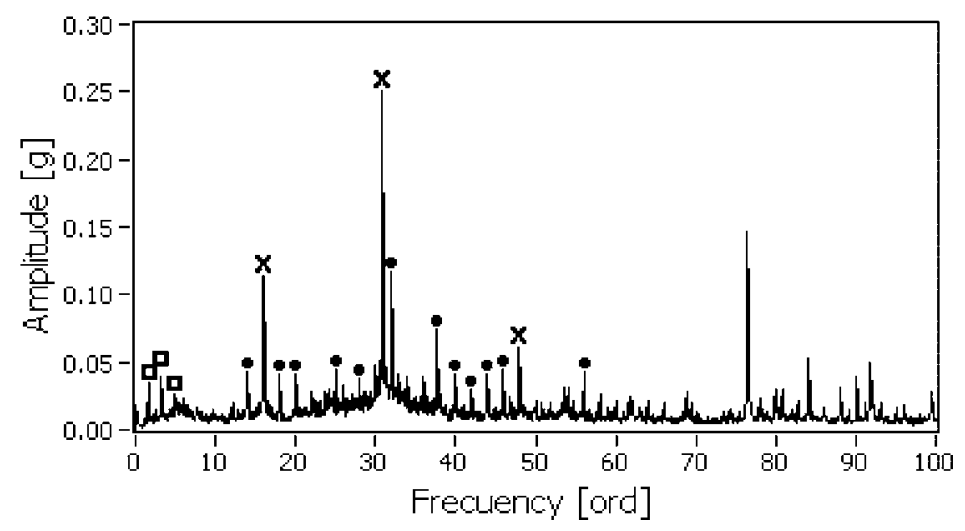

Fig. 22. Order Spectrum. Key: $\mathrm{x}=$ harmonics of GMF1, $\square=$ harmonics of GMF2, and $\bullet=$ harmonics of 2 ord.

dipper (loading cycle). Figure 14 shows the speed variations during the loading cycle of the dipper. We can notice the fast rotation speed changes during this cycle.

It can clearly be seen that in the order spectrum of Fig. 22 the harmonics order components of GMF1 and GMF2 indicated by the markers, remain in the same frequency position. The harmonics of 2 ord are due to the common factor between the number of teeth of the first two gears: $16=2 \cdot 8$ and $168=2 \cdot 84$. The gear mesh frequencies and their harmonics do not spread over spectral lines as is shown in Fig. 21 even though the rotation speed was significantly changing. This analysis then allows us to analyse the spectrum in a similar way as machinery operating at constant speed.

\section{Conclusions}

Computed order tracking can be used effectively in the vibration analysis of variable speed machines where spectral analysis using the standard FFT method, the most common basic technique used to diagnose machine problems, cannot be employed. Computed order tracking is a particularly attractive technique, because it is significantly simpler than the hardware solution, and data acquisition can be made with typical commercial data acquisition instrumentation.

The method's accuracy has been tested with various simulated signals. Simulation allows the determination of exact results, which were used as a base for carrying out comparisons. The noise was defined as the difference between the true signal and the resampled signal, and had the following sources:

1. Violation of the assumption that the angular shaft acceleration is constant.

2. Error in the detection of the pulse arrival times due to a low pulse sampling frequency.

3. Error in the estimation of the amplitude at resampled points due to a low signal sampling frequency.

In the first source of error, the user does not have any input, since the error only depends on factors such as the machine varying its speed. Nevertheless, the following practical conclusions are inferred from the results. If the acceleration changes value, but not sign, that is, the rotation speed always increases or decreases (as is the case in run-up or run-down procedures), then the error in the COT is insignificant. If the acceleration changes sign, then the error is significant. The most critical case is when the acceleration changes sign periodically. In this case, various spectral components that are multiples of the change in acceleration frequency appear in the order spectrum, and this can lead to a misinterpretation. The solution for increasing the accuracy of the method in this case is to increase the number of pulses per revolution.

The effect of the second source of error can be diminished by using a high sampling frequency. We define an acceptable error as one where the amplitude of the spectral component generated by the noise is in the order of magnitude of that due to the Hanning windowing effect. The pulse sampling frequency must be 32.5 times the pulse frequency. This value is independent of the interpolation methods used for estimating the resampled amplitudes. 

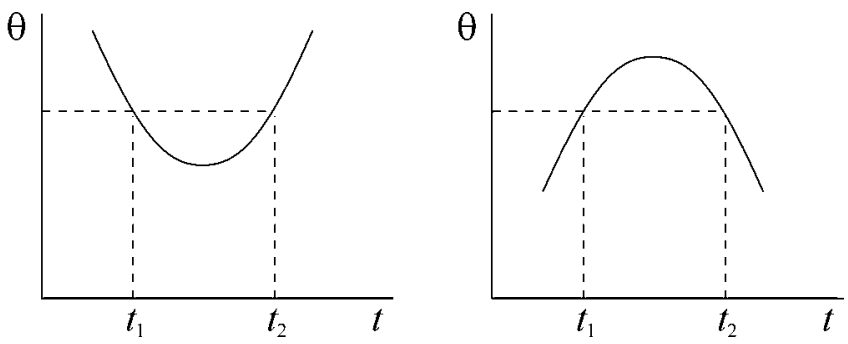

Fig. A1. Possible solutions for $t$.

In the third error source, there are two ways to increase the method's accuracy. The first is to use an appropriate sampling frequency in the signal acquisition for the different noise levels tolerated. The second way is to use a more sophisticated interpolation method in place of the linear interpolation method, such as the cubic spline interpolation. This improvement is only significant at low signal sampling frequencies.

The COT method was applied in the analysis of a vibration signal measured on a gearbox of a hoist transmission shovel in a copper mine. It has been shown that the method can be applied as an effective tool to carry out condition monitoring of these machines while they are working, and therefore avoid the high cost of lost production that occurs in traditional monitoring, where measurements are taken when the shovel is not in production mode. Condition monitoring of machinery working under variable speeds is not suitable using traditional spectral analysis techniques.

\section{Acknowledgment}

The authors would like to acknowledge FONDECYT of Chile, project No 1030323, for the financial support of this work.

\section{References}

[1] C.N. Tan and J. Mathew, Monitoring the vibrations of variable and varying speed gearboxes, The Institution of Engineers Australia, Vibration and Noise Conference, Melbourne 161-165, 1990.

[2] K.M. Boosley, R.J. McKendrick, C.J. Harris and C. Mercer, Hybrid computed order tracking, Mechanical Systems and Signal Processing 13 (1999), 627-641.

[3] K.R. Fyfe and E.D.S. Munck, Analysis of computed order tracking, Mechanical Systems and Signal Processing 11 (1997), $189-205$.

[4] J.K. Hammond and P.R. White, The analysis of non-stationary signals using time-frequency methods, Journal of Sound and Vibration 190(3) (1996), 419-447.

[5] P.N. Saavedra and F. Araya, Condition monitoring of variable-speed and load machinery using time-frequency distributions, Insight 43(8) (2001), 526-530.

[6] P.N. Saavedra and J.A. González, New revolution-order transform for analysing non-stationary vibrations, Insight 47(1) (2005), 29-35.

[7] J.R. Blough, Development and analysis of time variant discrete Fourier transform order tracking, Mechanical Systems and Signal Processing 17(6) (2002), 1185-1199.

[8] M.R. Bai, J. Jeng and C. Chen, Adaptive order tracking technique using recursive least-square algorithm, Transactions ASME Journal of Vibration and Acoustics 124 (2002), 502-511.

\section{Appendix A}

As discussed in Section 3.1.1, it is possible to calculate the time corresponding to each constant angle increment, $k \Delta \theta$, by solving Eq. (1) for $t$. Equation (1) always has two possible solutions for $t$, which are indicated in Fig. (A1).

$$
t_{k}=\begin{gathered}
-b_{1} \pm \sqrt{ } b_{1}^{2}-4 b_{2}\left(b_{0}-k \Delta \theta\right) \\
2 b_{2}
\end{gathered}
$$

Considering that the angle $\theta$ always increases with time, then the valid solution for $t$, is the one on the ascending side of the parabola. 

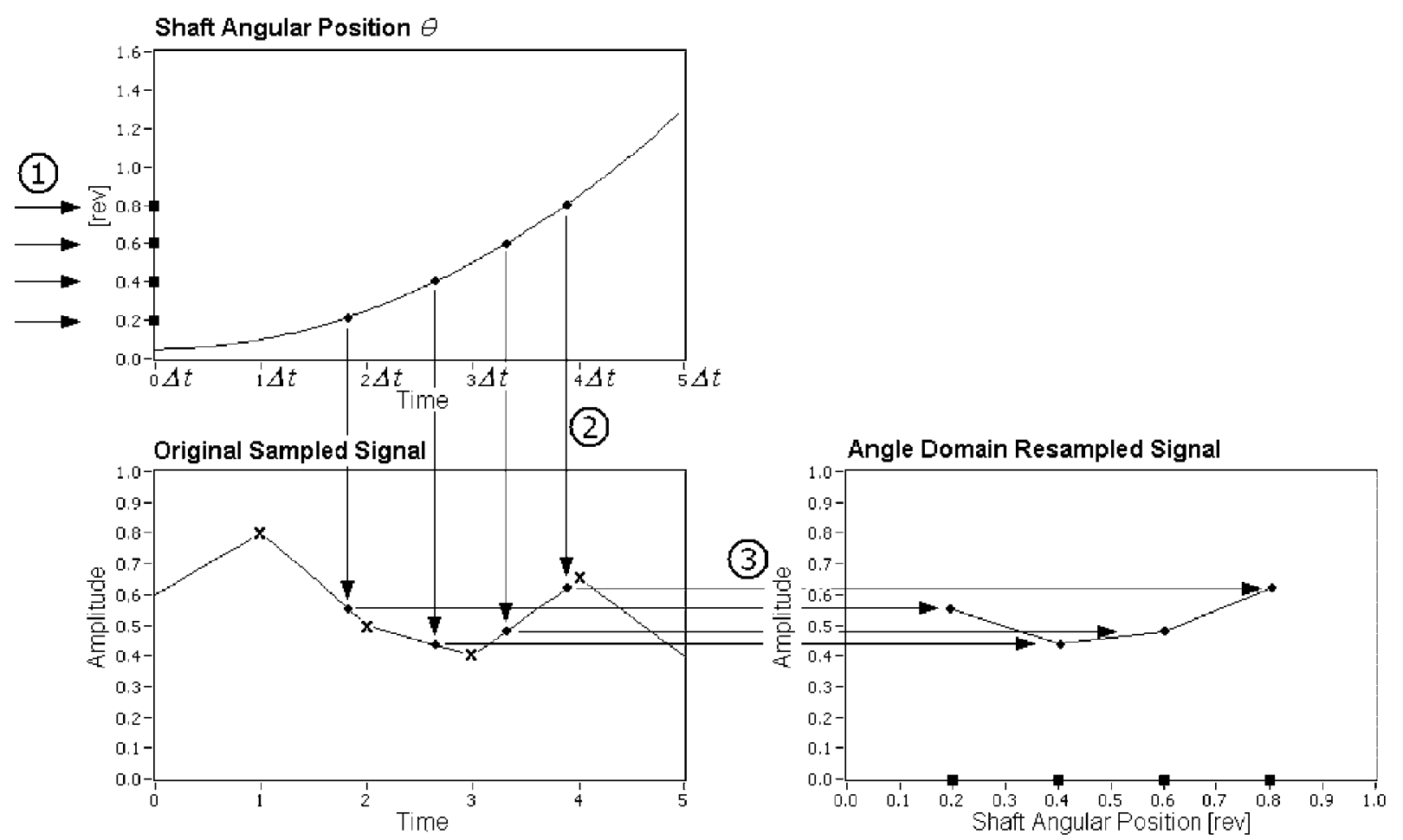

Fig. B1. Traditional method. Key: $\mathrm{x}=$ original sampled data, $\bullet=$ resampled data, and

$=$ constant angle increments

When the acceleration is positive, $b_{2}>0$ and the time corresponding to the ascending side of the parabola is the higher $t$ value, so that the sign considered in Eq. (A1) must be the positive one.

When the acceleration is negative, $b_{2}<0$ and the time corresponding to the ascending side of the parabola is the lower $t$ value, so that the sign considered in Eq. (A1) must be the positive one.

\section{Appendix B}

In the following figures it is illustrated the steps to get the resampled signal using the traditional method and the proposed method respectively, once the shaft angle position is determined.

Figure (B1) shows the different steps needed to be followed in the traditional method.

1) The angles, $\theta_{k}$, at constant angle increments, $\Delta \theta$, are defined (and indicated by arrow number 1 in Fig. B1).

2) The resample times, $t_{k}$, corresponding to each angle $\theta_{k}$, are determined from the quadratic polynomial of Eq. (1).

3) The amplitude, $x_{k}$, corresponding to the resample times, $t_{k}$, (indicated by arrow number 2 in Fig. B1), are calculated by interpolation between the original sampled data.

4) The resampled signal in the angle domain is constructed to obtain the previous values of the amplitude, $x_{k}$, (indicated by arrow number 3 in Fig. B1).

Figure (B2) shows the different steps needed to be followed in the proposed method.

1) The angles, $\theta_{n}$, at constant time increments, $\Delta_{t}$, are defined (and indicated by arrow number 1 in Fig. B2).

2) The angle domain signal is constructed: $x_{n}$ versus $\Theta_{n}$ corresponding to each original sampled data (indicated by arrow number 2 in Fig. B2).

3) The amplitude, $x_{k}$, corresponding to constant angle increments, $\theta_{k}$, (indicated by arrow number 3 in Fig. B2), are calculated by interpolation between the original sampled data $x_{n}$. 


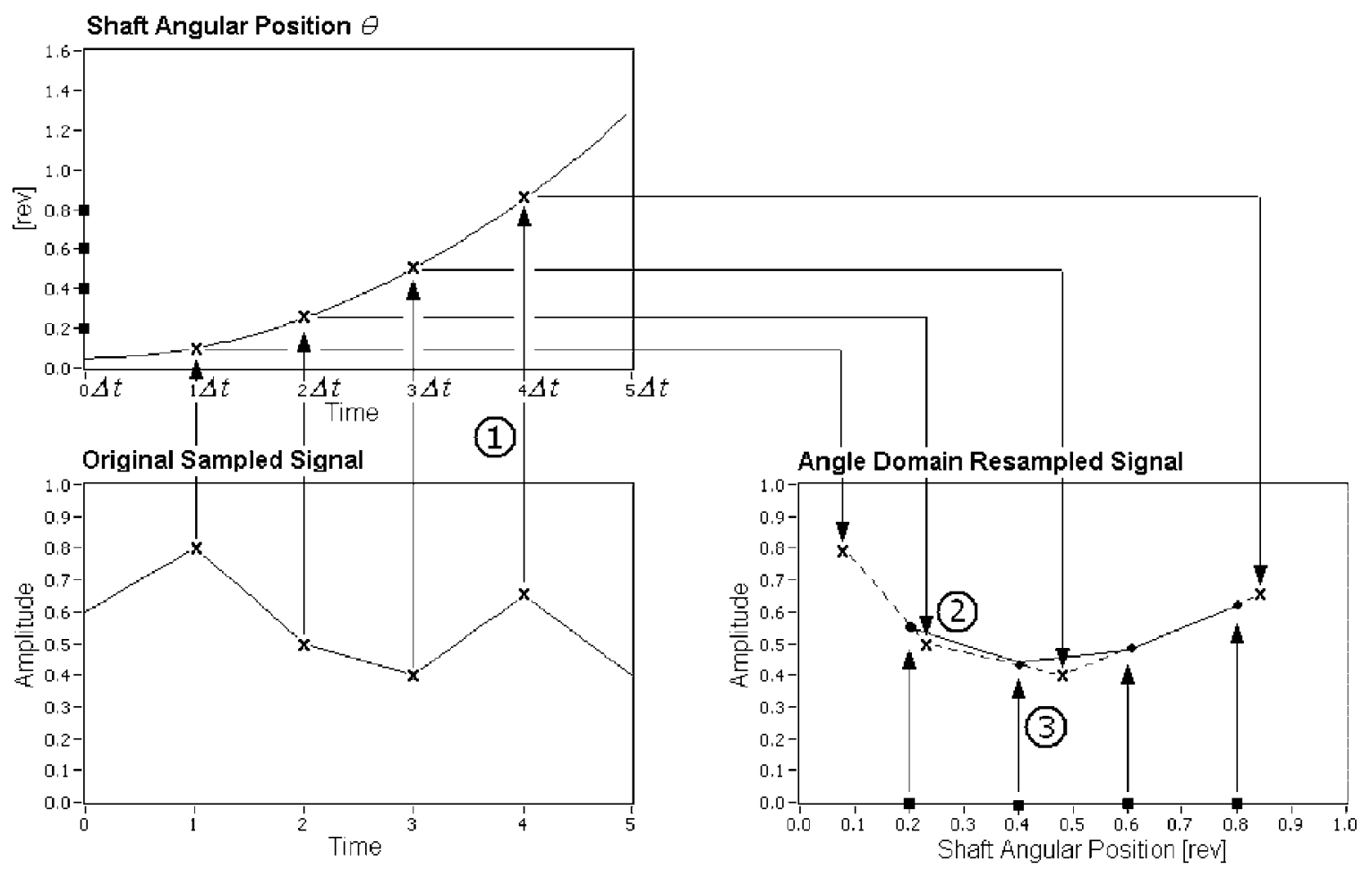

Fig. B2. Proposed method. Key: $\mathrm{x}=$ original sampled data, $\bullet=$ resampled data, and $\mathbf{D}=$ constant angle increments. 

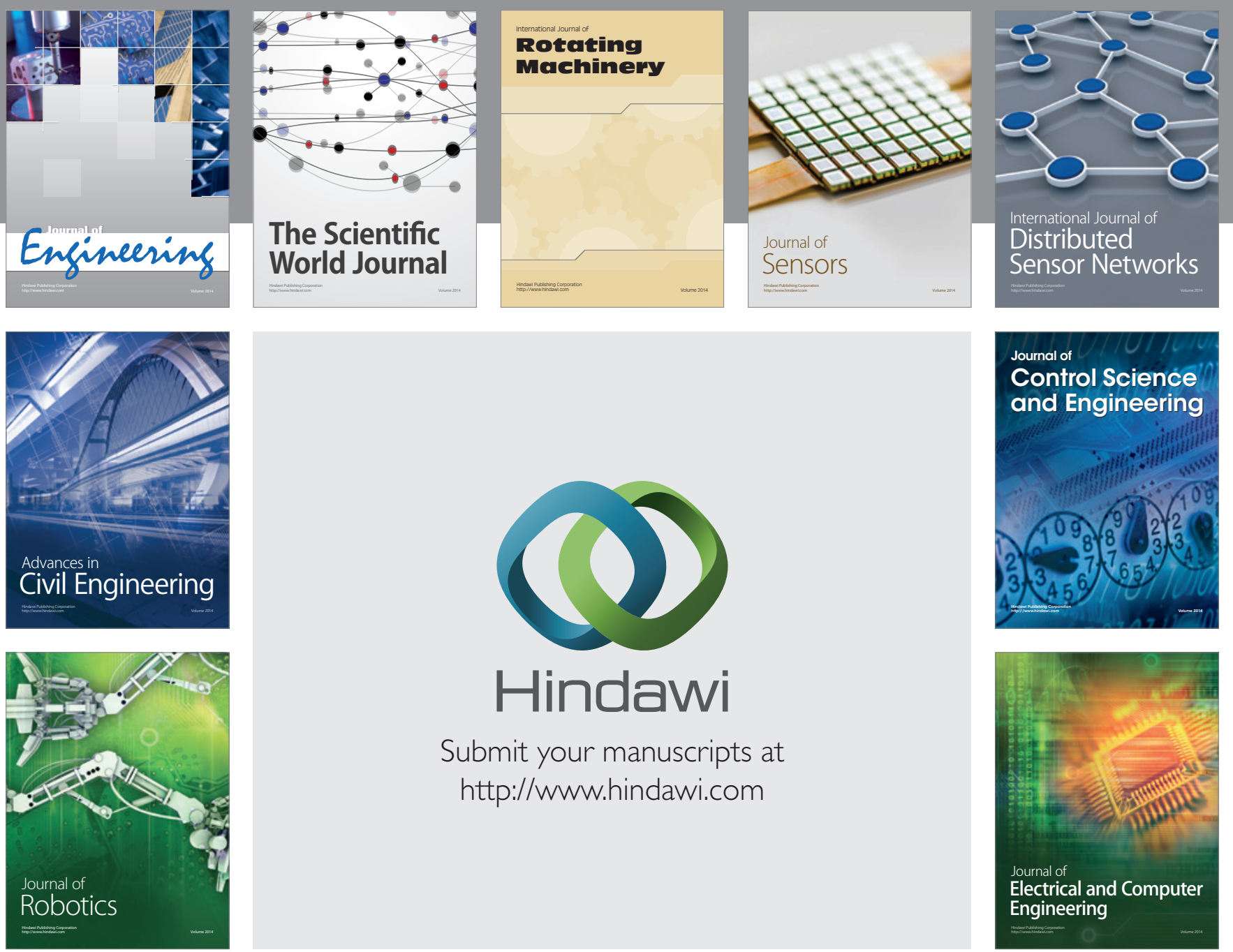

Submit your manuscripts at

http://www.hindawi.com
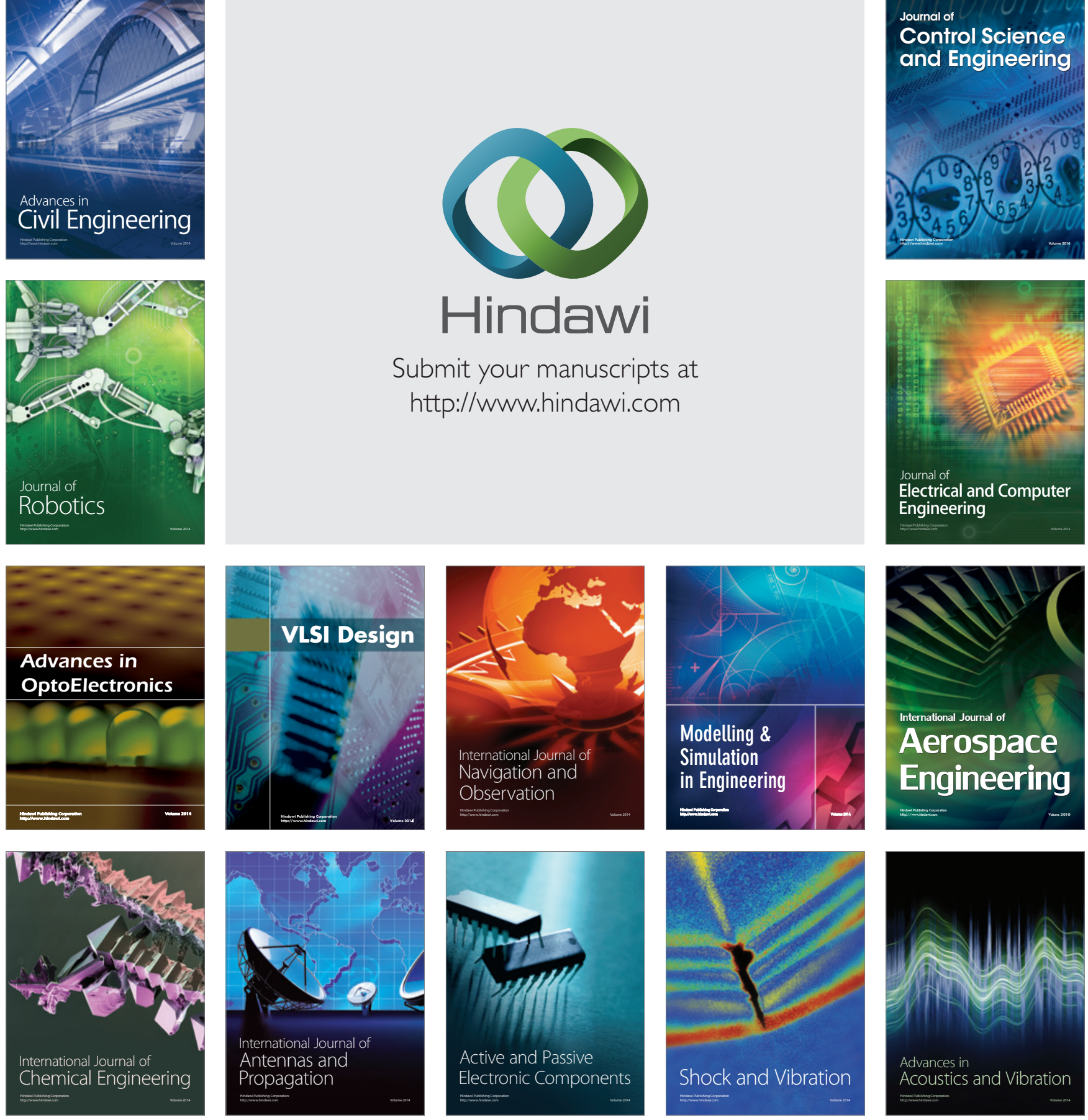